\title{
STUDIES OF THE COEFFICIENT OF VARIATION OF THE MAGNITUDE OF EEG SIGNALS
}

\author{
W. H. Round ${ }^{1}$, J. W. Sleigh ${ }^{2}$ \\ ${ }^{1}$ Department of Physics and Electronic Engineering, University of Waikato, Hamilton, New Zealand \\ ${ }^{2}$ Department of Anesthetics, Waikato Hospital, Hamilton, New Zealand
}

\begin{abstract}
An analysis of the variation in magnitude of EEG signals in various frequency bands of anesthetized patients and normal sleeping volunteers was carried out. The coefficient of variation $(\mathrm{CoV})$, i.e. the standard deviation/mean, within 10 second epochs was found to be quite constant throughout the whole of the EEG recordings and was typically about 0.46. This was found to be the case for both the patients and the volunteers. Histograms of the magnitudes indicated that the magnitudes are distributed as $f(x)=\beta x \mathrm{e}^{-\alpha x^{2}}$ functions. However a $\mathrm{CoV}$ of 0.46 is consistent with $f(x)=\beta x \mathrm{e}^{-\alpha x^{3}}$ functions. The non-stationary nature of the EEG is such that it is likely that while over short periods the EEG magnitudes are distributed as $f(x)=\beta x \mathrm{e}^{-\alpha x^{3}}$ functions, variations of $\alpha$ over time mean that in the long term the EEG magnitudes are distributed as $f(x)=\beta x \mathrm{e}^{-\alpha x^{2}}$ functions.
\end{abstract}

Keywords - EEG, coefficient of variation, anesthesia

\section{INTRODUCTION}

The EEG is has long been used as a diagnostic tool to analyze brain activity. Various models have been suggested to describe the way that the brain produces the EEG [e.g., 1]. The EEG has a chaotic nature and is produced by sources that are quite non-stationary, but such systems are often studied over sufficiently short time intervals that they may be considered stationary $[2,3]$. Here we analyze the magnitude of the EEG in terms of its covariance in various frequency bands.

\section{MethodolOGY}

EEG signals were recorded for 107 patients undergoing general anesthesia for surgery and 6 normal volunteers during normal sleep without anesthesia. The data was acquired with an Aspect A1000 EEG monitor. The EEG was sampled at 256 samples per second. No filtering was applied to the data during recording.

Spectrograms were calculated using the Fast Fourier Transform [4] for each of the EEG signals using a 256 point Hamming window with 128 point overlap of successive windows. This resulted in the spectrogram being calculated at 0.5 second intervals and in $1 \mathrm{~Hz}$ bands. No pre-filtering or artifact removal was applied to the data. The spectrogram's magnitude at a chosen frequency was divided up into tensecond epochs containing 21 samples of the magnitude. For each of these epochs the mean and standard deviation of the magnitude samples was calculated. Also, the coefficient of variation $(\mathrm{CoV})$ (i.e. the standard deviation/mean) was calculated.

\section{MAGNITUDE VARIATION AND ANAESTHETISED PATIENTS}

Graphs of these parameters for the $20 \mathrm{~Hz}$ frequency band of a patient undergoing general anesthesia are shown in Fig. 1. Also shown in Fig. 1 is the associated EEG and the spectrogram. In the spectrogram the darker regions represent the lowest activity, and the lighter regions the highest.
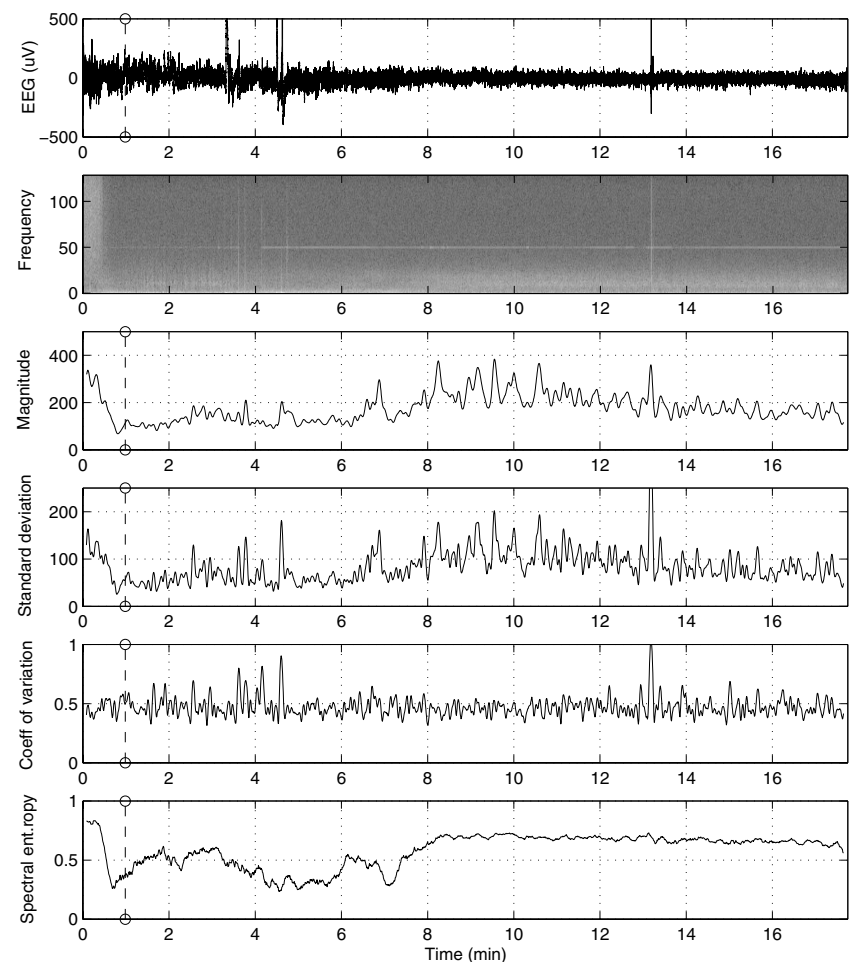

Fig. 1. The EEG and graphs of associated derived parameters for a patient undergoing general anesthesia. The top two graphs are the EEG and its associated spectrogram. The next three graphs are of (from top to bottom) the mean magnitude, the standard deviation and the coefficient of variation of ten second epochs for the $20 \mathrm{~Hz}$ band. The bottom graph is the spectral entropy of the EEG signal.

The vertical dashed line represents the time at which the anesthetist judged that the patient was unconscious. The 


\section{Report Documentation Page}

\begin{tabular}{|l|c|c|}
\hline $\begin{array}{l}\text { Report Date } \\
25 \text { Oct } 2001\end{array}$ & Report Type & Dates Covered (from... to) \\
\hline
\end{tabular}

Title and Subtitle

Studies of the Coefficient of Variation of the Magnitude of EEG

Signals

Author(s)

Performing Organization Name(s) and Address(es)

Department of Physics and Electronic Engineering University of

Waikato Hamilton, New Zealand

Sponsoring/Monitoring Agency Name(s) and Address(es)

US Army Research, Development \& Standardization Group

(UK) PSC 802 Box 15 FPO AE 09499-1500
Contract Number

Grant Number

Program Element Number

Project Number

Task Number

Work Unit Number

Performing Organization Report Number

Sponsor/Monitor's Acronym(s)

Sponsor/Monitor's Report Number(s)

\section{Distribution/Availability Statement}

Approved for public release, distribution unlimited

\section{Supplementary Notes}

Papers from 23rd Annual International Conference of the IEEE Engineering in Medicine and Biology Society, October 25-28, 2001, held in Istanbul, Turkey. See also ADM001351 for entire conference on cd-rom.

\begin{tabular}{|l}
\hline Abstract \\
\hline Subject Terms \\
\hline $\begin{array}{l}\text { Report Classification } \\
\text { unclassified }\end{array}$ \\
\hline
\end{tabular}

Classification of Abstract

unclassified

Number of Pages

5
Classification of this page

unclassified

\section{Limitation of Abstract}

UU 
data collection was terminated before the patient woke up again. In the spectrogram it is seen that the patient's EEG activity was initially high at all during the early stages of the data collection when the patient was awake. A collapsing of the activity at the higher frequencies as the patient went to sleep is also seen. Also seen in the EEG are large amplitude signals at approximately $3.5 \mathrm{~min}, 4.5 \mathrm{~min}$ and $13 \mathrm{~min}$. They are probably EMG artifacts, and they can also be detected in the spectrogram.

Also shown is a graph of the spectral entropy to act as an indicator of patient consciousness. It is noted that the spectral entropy decreased considerably some 20 seconds before the time that the anesthetist judged the patient lost consciousness, thus loss of consciousness may have occurred somewhat than was realized.

In the graph of the mean magnitude, the decrease in magnitude as the patient went to sleep can be clearly seen, and at this frequency the magnitude increased somewhat from about 9 minutes to 13 minutes which can also be seen in the spectrogram.

The similarity between the mean magnitude graph and the standard deviation graph should also be noted. They are similar in form with the peaks in the magnitude graph also being seen in the standard deviation graph. It should be noted that large amplitude artifacts in the EEG correspond to the larger peaks in the covariance graph. This is probably to be expected as the nature of the standard deviation is such that it will emphasize contributions to it from the values that differ most from the mean, and larger differences are expected to occur with the large amplitude artifacts.

Generally, though, the standard deviations values are just under 0.5 of the corresponding mean magnitude value. This is true for the entire duration of covariance graph, even during the initial part when the EEG activity is higher.

Consider now Fig. 2. In this figure, covariance graphs have been calculated for the EEG signal in Fig. 1, but now for $5,15,25,35,45$ and $55 \mathrm{~Hz}$ frequency bands. Often the covariance graph peaks in any one frequency band are also seen at other frequency bands. Also the covariance tends to generally be just less than 0.5 (the modal value is 0.46 ) for all frequencies. This suggests that if histograms of the magnitude values in different frequency bands are constructed, then they will possibly be of a similar form as the ratios of the mean and the standard deviation of each are similar.

To find the possible form of the histogram, histograms were constructed of the magnitudes for each frequency band over the entire time of the EEG recording. These are presented in Fig. 3 for the EEG waveform in Figs. 1 and 2.

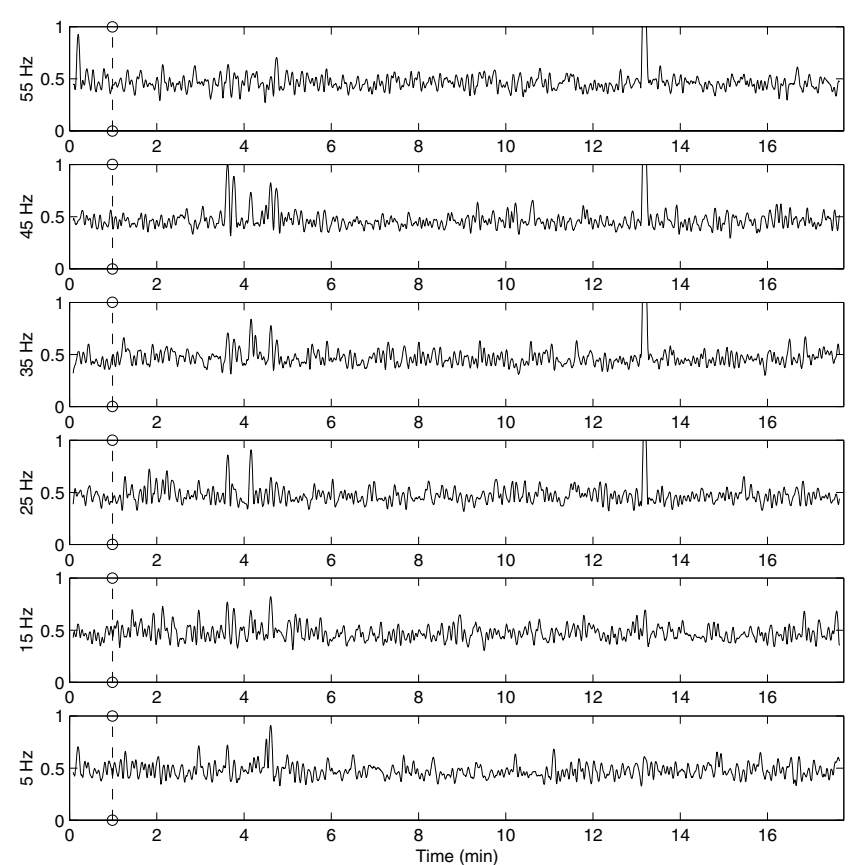

Fig. 2. Graphs of the covariance of the EEG signal in Fig 1. in different frequency bands.
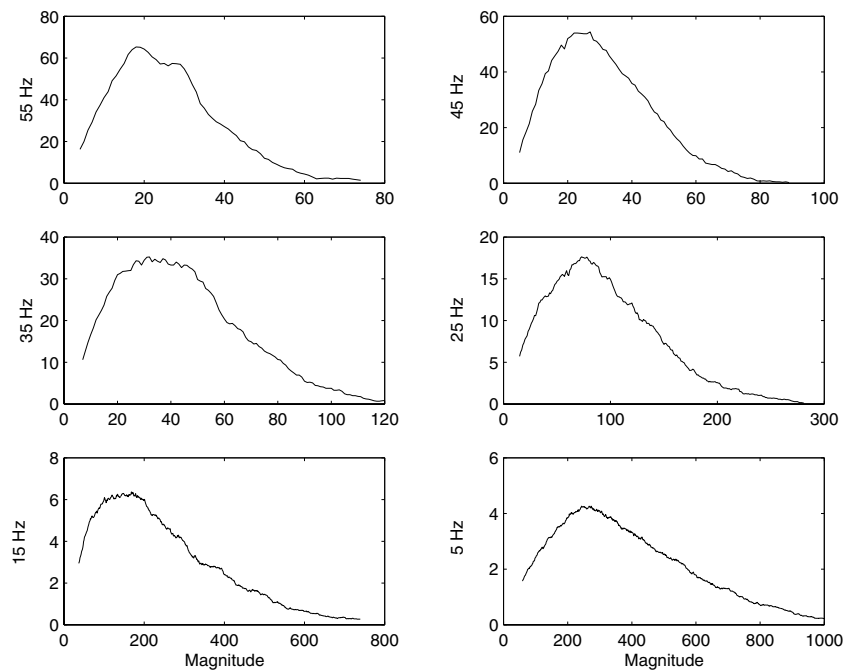

Fig. 3. Histograms of the magnitudes of the EEG waveform in Fig. 1 in various frequency bands.

The histograms do indeed have a similar form. The shapes of the histograms are such that they can be described by functions of the form

$$
f(x)=\beta x^{\mathrm{n}} \mathrm{e}^{-\alpha x^{\mathrm{m}}}
$$

where $x$ is the magnitude. When $x$ is small, the $x^{\mathrm{n}}$ term dominates and influences the initial rise of the histogram. For larger $x$, the $\mathrm{e}^{-\alpha x^{m}}$ term dominates and influences the later decrease in the histogram. $\alpha$ also influences the $x$ value at which the function reaches its maximum. $\beta$ determines the area of the histogram. 
Noting that for $\mathrm{n}>1$ that $f(x)$ is concave towards the right for low values of $x$ whereas for $\mathrm{n}=1$ the function is convex similar to the histograms in Fig. 3, it is reasonable to expect that $\mathrm{n}=1$.

For $\mathrm{n}=1$, and for low integer values of $\mathrm{m}$, the coefficients of variance of distributions described by such functions are

$$
\begin{array}{ll}
\mathrm{m}=1, & \mathrm{CoV}=0.7071 \\
\mathrm{~m}=2, & \mathrm{CoV}=0.5227 \\
\mathrm{~m}=3, & \mathrm{CoV}=0.4574
\end{array}
$$

For $\mathrm{n}=1$, then the covariance values indicate that for the typically observed covariance value of 0.46 that $m=3$. Thus the distributions should have histograms of the form

$$
f(x)=\beta x \mathrm{e}^{-\alpha x^{3}} .
$$

However coefficients of variation for the distributions in Fig. 3 are rather higher than 0.46, and are, on average, 0.62 . This indicates that

$$
1<\mathrm{m}<2
$$

rather than 3 .

Choosing $\mathrm{m}=2$ and fitting functions of the form $f(x)=x \mathrm{e}^{-\alpha x^{2}}$ to the histograms gives curves as in Fig. 4 .
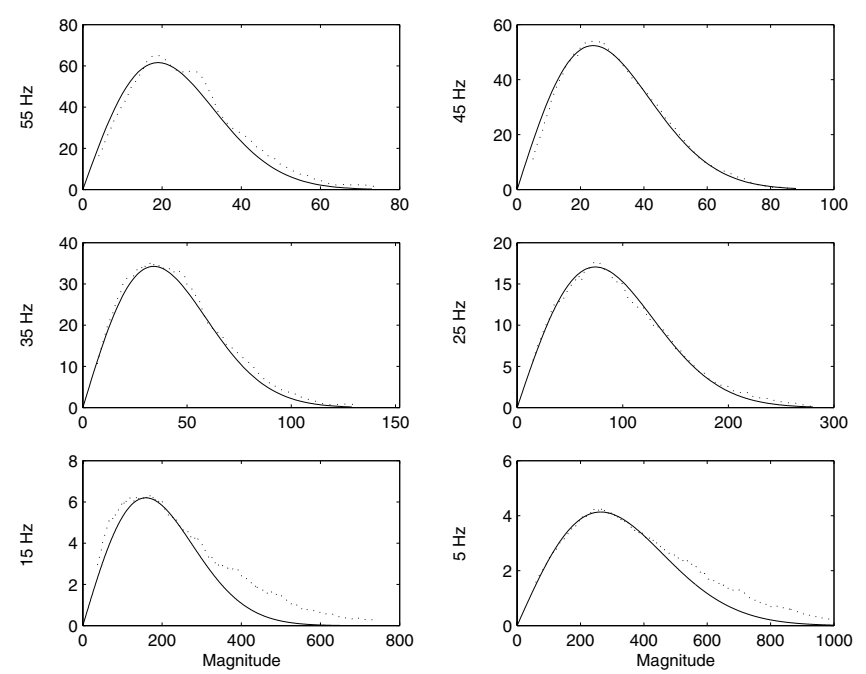

Fig 4. Dotted curves are corresponding graphs in Fig. 3., solid curves are fitted curves of the form $\beta x \exp \left(-\alpha x^{2}\right)$.

It is seen that the curves are generally well described by $f(x)=\beta x \mathrm{e}^{-\alpha x^{2}}$ curves except in the lower frequency bands. The differences are because the EEG is not that of a stationary system and there are longer-term variations in the magnitude at any frequency. Hence the histograms in Fig 4 are the summations of many shorter-term histograms. Shorter term histograms, if they could be formed with just 21 samples taken over 10 seconds as was done earlier, would reasonably be expected to be of the form $f(x)=$ $\beta x \mathrm{e}^{-\alpha x^{3}}$ and have, on average, coefficients of variation of 0.4574 .

At low frequencies where there is a lot of variation in the EEG magnitude (as can be seen in the spectrogram), the longer term histograms exhibit long tails from the histogram summations. At higher frequencies where there is not as much variation in the spectrogram and the system is more like a stationary system, the tail is not as pronounced. However it should be noted that the higher frequency bands' histograms exhibited two peaks: a small peak at large magnitudes due to the initial high activity before the patient went to sleep, and a large peak at small magnitudes when the patient was asleep. The smaller peak was excluded in fitting the functions to the histograms and calculating the coefficients of variation.

How well a summation of $\beta x e^{-\alpha x^{3}}$ functions can approximate a $\beta x \mathrm{e}^{-\alpha x^{2}}$ function is demonstrated in Fig. 5.

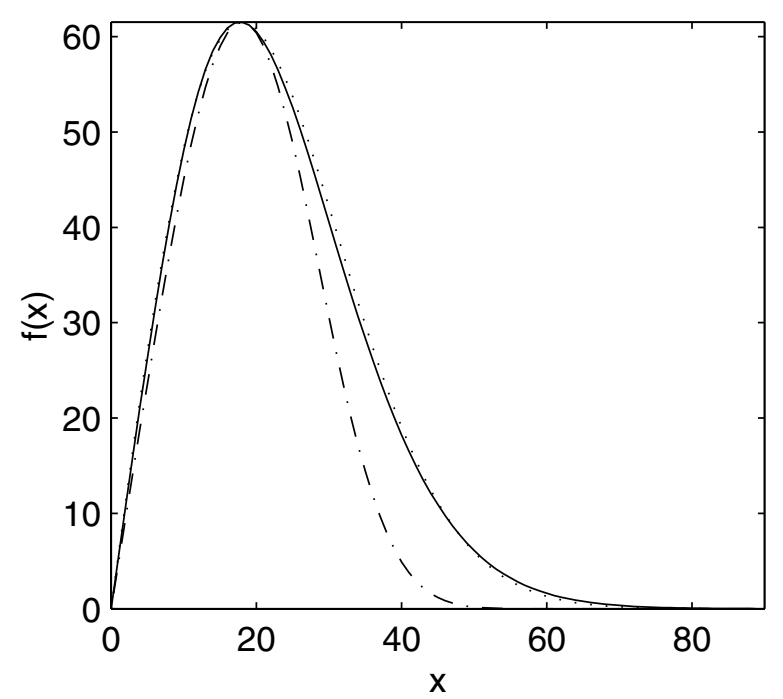

Fig. 5. The unbroken curve is generated from a summation a gaussian distribution of functions of the form $\beta x \exp \left(-\alpha x^{3}\right)$ chosen so that it is similar to the $55 \mathrm{~Hz}$ band curve in Fig 4. The dotted curve is a fitted curve of the form $\beta x \exp \left(-\alpha x^{2}\right)$, while the dot-dash curve is a fitted curve of the form $\beta x \exp \left(-\alpha x^{3}\right)$.

In Fig. 5, the solid curve is generated with from a sum of many $\beta x \mathrm{e}^{-\alpha x^{3}}$ functions with their peaks evenly distributed along the $x$-axis. The position of a peak is determined by the $\alpha$ value for each function. Their areas given by a gaussian curve centred at $x=18$ and with a standard deviation of 8 . These values, along with sum of their areas, are chosen so that the solid curve is similar to the curve fitted to the $55 \mathrm{~Hz}$ band data in Fig 4. The dotted and dot-dash curves are $\beta x \mathrm{e}^{-\alpha x^{2}}$ and $\beta x \mathrm{e}^{-\alpha x^{3}}$ functions respectively fitted to the unbroken curve so that their peaks coincide. Note that the $\beta x \mathrm{e}^{-\alpha x^{2}}$ curve is close fit while the $\beta x \mathrm{e}^{-\alpha x^{3}}$ curve isn't. If attempts are made to fit the $\beta x \mathrm{e}^{-\alpha x^{3}}$ curve's tail by altering $\alpha$ so that the rate of fall off is similar to the unbroken curve's, 
the curve moves considerably to the right and the fit is worse.

Over any time interval, the distribution of $\beta x \mathrm{e}^{-\alpha x^{3}}$ curves will not necessarily be just a simple gaussian distribution. If the EEG does not fluctuate in magnitude to any great extent, then it is not unreasonable for it to have such a distribution. However, if the magnitude does fluctuate significantly or is contaminated by artifact, then the distribution is unlikely to be gaussian or of any other simple function, but may have multiple peaks. In such a case it is unlikely to approximate a $\beta x \mathrm{e}^{-\alpha x^{2}}$ curve. At times when larger magnitude signals from artifacts combine with other shorter magnitude signals, the standard deviation of the magnitudes will increase more than the mean of the magnitudes, and thus the covariance will rise. Thus the covariance can be seen as an indicator of stationarity of the signal. The more it rises, the more the signal is fluctuating.

\section{MAGNITUDE VARIATION IN NORMAL SLEEP}

The coefficients of variation for 10 second epochs were calculated for the six volunteers. Graphs of these are shown in Fig. 6 for one of the volunteers, along with the spectral entropy. Note that during all sleep phases the covariance is again approximately 0.46 (modal values are $0.435,0.456$, $0.453,0.453,0.476$ and .459 respectively for the $55,45,35$, 25,15 , and $5 \mathrm{~Hz}$ bands) at all frequencies (although it does go significantly higher than this at certain times). This suggests that the histograms of the coefficients of variation at the different frequencies have the shape of $\beta x \mathrm{e}^{-\alpha x^{3}}$ functions for persons in normal sleep.

\section{DISCUSSION}

The data and analysis reported here for two EEG recordings is typical of all those studied. It seems quite surprising that the CoV of the EEG is so similar throughout all of the EEG recordings, and also that it is so similar at all frequencies. For the patients undergoing general anesthesia and for the patients in normal sleep, in the short term it tends to be approximately 0.46 both when they are fully asleep, when they are quite awake and at various stages in between. This suggests that if the magnitude in any frequency band is sampled, the samples are likely have a distribution which is similar in all frequency bands regardless of the state of consciousness of the subject. We have suggested here a possible form for the distribution, but note that over long periods of time the signal changes as the system that generates the EEG is non-stationary. Thus the distribution will alter and the function will change, but it can still be described by a function of the same basic form.

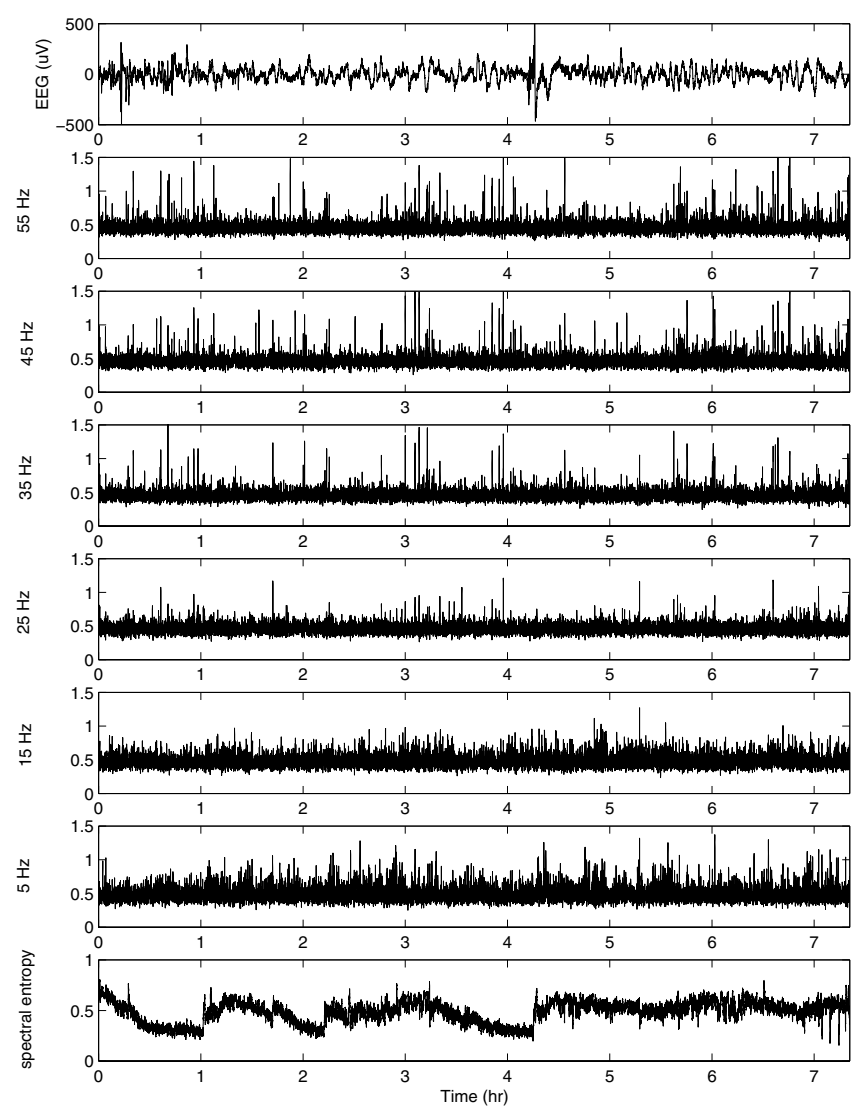

Fig 6. The EEG and graphs of the coefficient of variation of ten second epochs for the various frequency bands for a volunteer in normal sleep. The bottom graph is the spectral entropy of the EEG signal.

\section{REFERENCES}

[1] M. L. Steyn-Ross, D.A. Steyn-Ross, J.W. Sleigh and D.T.J. Liley, "Theoretical electroencephalogram stationary spectrum for a white-noise-driven cortex: Evidence for a general anesthetic-induced phase transition", Phys. Rev. E., vol. 60, no. 6, pp. $7299-7311,1999$.

[2] A. Cohen, Biomedical Signal Processing, CRC Press, 1986.

[3] S. Blanco, C. E. D’Attellis, S. I. Isaacson, O. A. Rosso, and R. O. Sine, "Time-frequency analysis of electroencephalogram series. II. Gabor and Wavelet Transforms", Phys. Rev. E, vol. 54, no. 6, pp. $6661-6672,1996$.

[4] J. W. Cooley, and J. W. Tukey, "An algorithm for the machine computation of the complex fourier series", Math. Comp., vol. 19, pp. $297-301,1965$. 